University of Wollongong

Research Online

Faculty of Social Sciences - Papers (Archive) Faculty of Arts, Social Sciences \& Humanities

$1-1-2012$

Selective imitation in 6-month-olds: the role of the social and physical context

Sabine Seehagen

Ruhr-Universität Bochum, University of Sheffield

Jane S. Herbert

University of Sheffield, herbertj@uow.edu.au

Follow this and additional works at: https://ro.uow.edu.au/sspapers

Part of the Education Commons, and the Social and Behavioral Sciences Commons

Research Online is the open access institutional repository for the University of Wollongong. For further information contact the UOW Library: research-pubs@uow.edu.au 


\title{
Selective imitation in 6-month-olds: the role of the social and physical context
}

\author{
Abstract \\ Six-month-old infants' learning of a new action from two different models (mother/stranger) was \\ assessed in two settings (home/laboratory). In the laboratory, a significant number of infants learned the \\ action from a stranger but not from their mother. In the infants' homes, this pattern was reversed. \\ Keywords \\ context, physical, role, 6-month-olds:, imitation, social, selective \\ Disciplines \\ Education | Social and Behavioral Sciences \\ Publication Details \\ Seehagen, S. \& Herbert, J. S. (2012). Selective imitation in 6-month-olds: the role of the social and \\ physical context. Infant Behavior and Development, 35 (3), 509-512.
}




\title{
Selective imitation in 6-month-olds: The role of the social and physical context
}

\author{
Sabine Seehagen ${ }^{1,2}$ and Jane S. Herbert ${ }^{1}$ \\ ${ }^{1}$ University of Sheffield, Sheffield, UK \\ ${ }^{2}$ Ruhr-Universität Bochum, Bochum, Germany
}

Key words: Infant, Learning, Imitation, Maternal, Context

Running head: Selective imitation

Authors' Notes

This research was supported by a University of Sheffield studentship and a Wellcome Trust VIP Bridging Award to Sabine Seehagen. It composed a portion of the doctoral dissertation of Sabine Seehagen and was completed under the supervision of Jane Herbert. The authors would like to thank Andy Ham for stimuli construction and all of the infants and parents who participated in this project. A fuller report of the experiment will be provided upon request. Corresponding author: Dr Sabine Seehagen, Fakultät für Psychologie, Ruhr-Universität Bochum, Universitätsstr. 150, 44780 Bochum, Germany. Phone: +49 (0)234 322 8537; Fax: +49 (0)234 320 3169; E-mail: sabine.seehagen@rub.de. 


\begin{abstract}
Six-month-old infants' learning of a new action from two different models (mother/stranger) was assessed in two settings (home/laboratory). In the laboratory, a significant number of infants learned the action from a stranger but not from their mother. In the infants' homes, this pattern was reversed.
\end{abstract}


In naturalistic settings, observing and copying another person's actions is an important way in which infants acquire new skills and behaviours (Barr \& Hayne, 2003). Capitalizing on this natural tendency, standardized imitation procedures assess learning and memory by presenting the infant with a model who demonstrates a series of actions with objects, and measuring the infant's ability to reproduce these actions afterwards (e.g., Herbert \& Hayne, 2000; Meltzoff, 1985, 1988). These standardized procedures are widely used to document age-related changes in learning and memory (for a review, see Hayne, 2004).

In the vast majority of imitation studies, the model demonstrating the actions to the infant is an unfamiliar adult. Recently, however, research has begun to examine whether the relationship between the model and the infant may impact on learning and memory (e.g., Devouche, 2004; Ryalls, Gul, \& Ryalls, 2000; Seehagen \& Herbert, 2010, 2011). For example, peers have been shown to be a particularly effective model for infants who are older and have had extended regular peer contact via nursery or playgroup (Seehagen \& Herbert, 2011). It is less clear, however, whether the level of familiarity the infant has with an adult model has an impact on the model's effectiveness.

A few studies have compared imitation from mothers and unfamiliar adults, and their findings suggest that there might be no general advantage in a mother demonstration compared to a stranger demonstration (e.g., Devouche, 2004; Seehagen \& Herbert, 2010). It is important to note, however, that these studies focused on infants who were 8 months and older. Since infants start displaying stranger anxiety from about $7-8$ months of age (Waters, Matas, \& Sroufe, 1975), it is possible that emotional factors might be overriding any potential differences in the effectiveness of adult models with differing levels of familiarity to the infant. For example, being wary of a stranger might lead to lower imitation scores after demonstrations from this model due 
to infants' unwillingness, rather than their inability, to carry out the target action. Thus, wariness of strangers might sometimes lead to an underestimation of an unfamiliar model's effectiveness in older infants. Thus, further studies are needed to compare imitation from a familiar versus unfamiliar model at an age when stranger anxiety is not yet displayed. In addition to the social context (i.e., the model) occurring within the imitation task, familiarity with the physical context (i.e., the setting) impacts on infants' learning and memory performance (Hayne, Boniface, \& Barr, 2000; Jones \& Herbert, 2008). For example, being overly familiar with an environment can be detrimental to the flexible expression of memory in imitation tasks (Jones \& Herbert, 2008). Twelve-month-old infants who experienced a unique physical context for encoding and retrieval exhibited imitation when they were tested 10 min later in a generalization task with related stimuli. In contrast, infants for whom the context was not unique to the learning and test session (because they had also experienced the context prior to the demonstration session and/or during the retention interval) did not exhibit imitation. Thus, an infant's previous history with a learning environment can impact on his or her learning and memory performance.

While both the social and physical context appear to be important influences on infant learning and memory, it is unlikely that they act independently from each other. Instead, infants might take into account both the physical and social context of a situation when learning new information (e.g., Stenberg, 2009; Stenberg \& Hagekull, 2007). For example, when encountering an ambiguous situation in an unfamiliar environment, 12-month-old infants are prone to paying attention to an unfamiliar adult rather than their own mother (Stenberg \& Hagekull, 2007), suggesting they might perceive an unfamiliar person as a particularly valuable source of information in a novel environment. It is unknown whether younger infants might already have some basic assumptions about certain models being particularly useful or trustworthy as teachers in certain settings. 
The current experiment examined whether 6-month-old infants' imitation varied as a function of the relationship between the model (mother or unfamiliar female) and the physical context in which learning occurs (home or unfamiliar lab). Specifically, we predicted that infants would be most likely to copy the action of a model who they might expect to provide useful information in a particular physical context (Stenberg \& Hagekull, 2007) i.e., a familiar model in a familiar context and an unfamiliar model in an unfamiliar context.

The final sample consisted of 84 infants (48 females) who were 6 months old (+/- 10 days). The majority of infants (95\%) were Caucasian ( $71 \%$ of mothers reporting) and came from families with a modest to high socio-economic background. Nine additional infants were excluded due to crying $(n=3)$, failure to touch the stimulus $(n=1)$, experimenter error $(n=2)$, a phone call interrupting the demonstration at an infant's house $(n=1)$, and maternal verbal or physical prompting of the target actions $(n=2)$.

The stimulus was a decorated wooden box $(37 \mathrm{~cm}$ high, $24 \mathrm{~cm}$ wide, $15 \mathrm{~cm}$ deep) similar to that used in Herbert, Gross and Hayne (2006). The front of the box was painted with a frog's face and was flat, with the exception of two red protruding eyes in the middle of the box $(5 \mathrm{~cm}$ in diameter) and two small wooden bees $(2 \mathrm{~cm} \mathrm{x} 1 \mathrm{~cm})$ attached near the top. A $7.7 \mathrm{~cm}$ high and $11 \mathrm{~cm}$ deep white wooden base was attached to the front of the box. A white wooden button (diameter $6.5 \mathrm{~cm}$ ) was recessed into the centre of the base. The middle of the button was marked by a round piece of blue fabric (diameter $1 \mathrm{~cm}$ ). Pushing the button produced a frog-appropriate croaking sound for approximately $3 \mathrm{sec}$.

Half the infants $(n=42)$ were tested in the University of $x x$ infant lab and the other half were tested in their own homes. During a short warm-up phase, the experimenter interacted with the infant until he or she appeared to be comfortable. In each testing context (home and lab) 
infants were randomly assigned to one of two demonstration groups or a baseline control group $(n=14)$. All sessions were video-recorded.

Experimenter Demonstration. The infant was seated on the floor with his or her mother next to or immediately behind. The experimenter sat on the floor opposite the infant and demonstrated the target action of pushing the button with her hand, causing the frog to make a croaking noise. The target action was demonstrated six times in succession, out of the infant's reach. The stimulus and the target action were not verbally described or labelled and the mother was asked to refrain from any commenting or directing the infant. The experimenter used empty narration to maintain the infant's attention to the demonstrations e.g., "Look" or "What's this?" After the last demonstration, the experimenter immediately positioned the stimulus within reach in front of the infant. Each infant was given a $20 \mathrm{sec}$ response period (see Meltzoff, 1985) in which to reproduce the target action from the time he or she first touched the object. Production of the target action was not verbally or physically prompted.

Mother Demonstration. The procedure for this group was identical to that of the Experimenter group with the exception that each infant's own mother demonstrated the target action and conducted the test while the experimenter sat behind or beside the infant. Prior to the demonstration, mothers were trained how to demonstrate the target action by watching a silent video out of view of the infant. The experimenter explained to each mother that she should only use empty narration during the demonstration. All but one mother in this group adhered to the procedure, and this mother's infant was excluded from the sample.

Baseline. Infants in this group did not observe a demonstration of the target action. Instead, their spontaneous production of the target action was assessed. The test session for this group was identical to that used in the demonstration groups. Previous research (Meltzoff, 1988) has shown that baseline control groups, where infants do not see the stimuli before the test, 
provide a more conservative measure of the spontaneous production of actions than control groups where the experimenter touches or manipulates the test stimuli during the "demonstration" session without performing the target actions.

One observer scored the presence or absence of the target action (pressing the button to produce the sound) during test from videotape. A second independent coder scored the performance of $50 \%$ of the sample. Inter-rater reliability was $\kappa=.93$.

Latency scores: To determine whether there was an effect of condition on infants' willingness to interact with the stimulus, we coded the latencies of their first touch of the stimulus from the time it was placed within their reach. There was no significant difference in infants' latency scores as a function of group, $F(5,78)<1$. Thus, infants' motivation to start interacting with the stimulus did not differ across settings and models.

Imitation scores: Data analysis was conducted in the same way as in Herbert et al. (2006). To assess whether there were any differences in the numbers of infants who performed the target action as a function of group in each location, $\chi^{2}$-tests were conducted. Significant group differences were further analysed using Fisher's Exact Test. Imitation was evidenced if more infants in a demonstration group performed the target action than in the baseline group.

Imitation in the laboratory context. Significantly more infants in the Experimenter group $(n=5)$ than in the Baseline group $(n=0)$ performed the target action, $\left(\chi^{2}(1, N=28)=6.09, p=\right.$ .01 ; Fisher's Exact Test (one-sided), $p=.02$ ). In contrast, although approaching significance, the number of infants performing the target action in the Mother group $(n=3)$ did not differ significantly from baseline $\left(\chi^{2}(1, N=28)=3.36, p=.07\right)$.

Imitation in the home context. A significant number of infants exhibited imitation in the Mother group $(n=5)$, compared to in the Baseline group $(n=0), \chi^{2}(1, N=28)=6.09, p=.01$; Fisher's Exact Test (one-sided), $p=.02$ ). In contrast, although there was a trend towards more 
infants imitating the target action in the Experimenter group $(n=3)$ than in the Baseline group, the differences did not reach conventional levels of significance, $\left.\chi^{2}(1, N=28)=3.36, p=.07\right)$.

This experiment revealed that both the social and the physical context can influence 6month-old infants' learning of a novel action. When tested in an unfamiliar environment, infants who had observed an unfamiliar model exhibited imitation. When tested in a familiar environment, infants who had observed a familiar model exhibited imitation. In other words, infants required a match of familiarity between the physical and social context for significant levels of imitation to occur. Previous research with older infants failed to find a significant difference in imitation from familiar and unfamiliar adults in a laboratory setting (e.g., Devouche, 2004; Seehagen \& Herbert, 2010). Put differently, mothers have repeatedly demonstrated their effectiveness as teachers in an unfamiliar setting, at least with older infants. Thus, it is unlikely that our results simply reflect mothers being "bad" models in the lab and the experimenter being a "bad" model in the infants' homes. Instead, our results likely reflect age-related differences in infants' use of differently familiar teachers in different settings.

Strouse and Troseth (2008) suggested that infants might develop expectations about whether certain situations are designed for learning or for other purposes. In the present experiment, 6-month-old infants may have had expectations about the two models' usefulness as teachers. During the first few months of life, infants tend to spend the largest parts of their days together with their mothers at home, at least in Western societies. During this time, they interact with their mother and might start associating their mother and her actions with the familiar environment. In other words they learn "what goes with what and what happens where" (RoveeCollier, 1996, p. 386). After 6 months of experience, infants are clearly capable of profiting from interactions with their mothers, and have become used to her as a valuable source of information in the familiar setting of the own home. However, they might yet have to learn to use their 
mother in the same way in a completely novel environment. Conversely, an infant might have experienced unfamiliar people mostly in unfamiliar settings and thus have formed the expectation that unfamiliar people are in charge in unfamiliar environments. This way, young infants might more readily make use of a particular model for learning when they are encountered in the "right" physical context.

Our results suggest that infants' ability to learn novel behaviours in different situations might depend on their previous learning history and expectations about a model and a setting. In future studies it will be important to document infants' everyday social experiences (e.g., day care attendance) as well as their development in other domains (e.g., onset of stranger anxiety) longitudinally and to relate these changes to the emergence of imitative abilities in different social and physical contexts.

\section{References}

Barr, R., \& Hayne, H. (2003). It's not what you know, it's who you know: Older siblings facilitate imitation during infancy. International Journal of Early Years Education, 11, 7-21.

Devouche, E. (2004). Mother versus Stranger: A Triadic Situation of Imitation at the End of the First Year of Life. Infant and Child Development, 13, 35-48.

Hayne, H. (2004). Infant memory development: Implications for childhood amnesia. Developmental Review, 24, 33-73.

Hayne, H., Boniface, J., \& Barr, R. (2000). The development of declarative memory in human infants: Age-related changes in deffered imitation. Behavioral Neuroscience, 114, 77-83. Herbert, J., Gross, J., \& Hayne, H. (2006). Age-related changes in deferred imitation between 6 and 9 months of age. Infant Behavior and Development, 29, 136-139. 
Herbert, J., \& Hayne, H. (2000). The ontogeny of long-term retention during the second year of life. Developmental Science, 3, 50-56.

Jones, E. J., \& Herbert, J. S. (2008). The effect of learning experiences and context on infant imitation and generalization. Infancy, 13, 596-619.

Meltzoff, A. N. (1985). Immediate and deferred imitation in fourteen- and twenty-four-month-old infants. Child Development, 56, 62-72.

Meltzoff, A. N. (1988). Infant imitation and memory: Nine-month-olds in immediate and deferred tests. Child Development, 59, 217-225.

Rovee-Collier, C. (1996). Shifting the focus from what to why. Infant Behavior and Development, 19, 385-400.

Ryalls, B. O., Gul, R. E., \& Ryalls, K. R. (2000). Infant imitation of peer and adult models: Evidence for a peer model advantage. Merrill-Palmer Quarterly: Journal of Developmental Psychology, 46, 188-202.

Seehagen, S., \& Herbert, J. S. (2010). The role of demonstrator familiarity and language cues on infant imitation from television. Infant Behavior and Development, 33, 168-175.

Seehagen, S., \& Herbert, J. S. (2011). Infant imitation from televised peer and adult models. Infancy, 16, 113-136.

Stenberg, G. (2009). Selectivity in infant social referencing. Infancy, 14, 457-473.

Stenberg, G., \& Hagekull, B. (2007). Infant looking behavior in ambiguous situations: Social referencing or attachment behavior? Infancy, 11, 111-129.

Strouse, G. A., \& Troseth, G. L. (2008). "Don't try this at home": Toddlers' imitation of new skills from people on video. Journal of Experimental Child Psychology, 101, 262-280. 
Waters, E., Matas, L., \& Sroufe, L. (1975). Infants' reactions to an approaching stranger:

Description, validation, and functional significance of wariness. Child Development, 46, 348-356. 